\title{
Adaptación y validación del cuestionario de interés y conocimiento en arte de Viena (VAIAK)
}

Adaptation and validation of the Vienna art interest and art knowledge

questionnaire (VAIAK)

\author{
Federico Martín González*
}

Débora I. Burin**

\section{Resumen}

La experticia mostró ser una variable relevante a la hora de estudiar procesos de recepción de arte visual. Con un enfoque psicométrico, el cuestionario de Conocimiento e Interés en Arte de Viena (VAIAK) se concibió como una medida unificada y validada de experticia. El objetivo del presente trabajo fue traducir, adaptar y validar el cuestionario para su uso en investigación con población argentina. 153 estudiantes completaron el cuestionario de manera grupal o individual de manera voluntaria. Se realizaron análisis de consistencia interna, de validez de constructo por medio de análisis factorial, y de validez discriminante a través de comparaciones entre estudiantes de psicología e historia del arte, así como correlación de medidas. Se obtuvo evidencia de la fiabilidad y la validez de las escalas de Interés, y de una parte adaptada de la escala de Conocimiento.

Palabras claves: Artes visuales, Conocimiento previo, Interés
Expertise proved to be a relevant variable when it came to study visual art reception processes. The Vienna Art Interest and Art Knowledge Questionnaire was conceived as an unified and validated measure of expertise using a psychometric focus. The objective of this study was to translate, adapt and validate the questionnaire for use in research with the Argentine population. 153 students voluntarily completed the questionnaire as a group or individually. Analysis of internal consistency, construct validity through factor analysis, and discriminant validity were performed through comparisons between psychology and art history students, as well as correlation of measures. Evidence was obtained of the reliability and validity of the Interest scales, and of an adapted part of the Knowledge scale.

Keywords: Visual arts, Prior knowledge, Interest

* Universidad de Buenos Aires. Facultad de Psicología. Instituto de Investigaciones, Buenos Aires, Argentina. Mail de contacto: fmgonzalez@psi.uba.ar

** Universidad de Buenos Aires. Facultad de Psicología. Instituto de Investigaciones ; Consejo Nacional de Investigaciones Científicas y Técnicas, Buenos Aires, Argentina. DOI: https://doi.org/10.46553/RPSI.16.32.2020.p68-78

Fecha de recepción: 28 de abril de 2020 - Fecha de Aceptación: 26 de octubre de 2020 


\section{Introducción}

El abordaje empírico en el estudio de la percepción de objetos artísticos tiene una larga tradición dentro del campo de la psicología (ej. Fechner, 1876; Arnheim, 1965), pero lejos de ser un área en vías de extinción tuvo un desarrollo reciente a la luz de nuevos modelos cognitivos y neurocognitivos (Pelowski, Markey, Forster, Gerger, \& Leder, 2017). Incluso en campos de estética filosófica se empezó a considerar el conocimiento producido desde la ciencia cognitiva como válido para enriquecer la comprensión de los fenómenos de recepción artística (Meskin, Robson, Ichino, Goffin, \& Monseré, 2018). Más allá del estudio básico de la conducta estética humana, el potencial rol de intervenciones basadas en obras de arte para la promoción de salud y bienestar (Fancourt \& Finn, 2019) y de conductas y actitudes prosociales (Kou, Konrath, \& Goldstein, 2019), constituye una motivación para entender los procesos de percepción y experiencia estética de obras de arte visual.

Entre las variables involucradas en los procesos de percepción y experiencia estética, varias investigaciones recientes se han centrado en dos de ellas debido a su impacto: el conocimiento previo y el interés. Tradicionalmente el estudio de la percepción artística se enfocó en las características del estímulo, como la composición, la simetría y el color. Pero las propiedades objetivas del estímulo, aunque relevantes y aún en proceso de estudio (por ejemplo, Ortlieb \& Carbon, 2019), son insuficientes para una comprensión adecuada de los fenómenos artísticos y la multiplicidad de respuestas que se dan frente a las obras. Una concepción generalmente consensuada del arte considera la convivencia de aspectos visuales con aspectos conceptuales, simbólicos o de significado. Factores del procesamiento llamado descendente o top-down, como la experiencia, el conocimiento, la expectativa o el interés, tienen efectos tanto sobre el procesamiento de aspectos visuales -patrones aprendidos de rastreo visual, por ejemplocomo sobre significados, conceptos o juicios valorativos diversos (Bullot \& Reber, 2013).

\section{Interés y conocimiento previo en la percepción de arte}

Dentro de los factores que hacen al procesamiento descendente, uno de los principales es la denominada experticia, que generalmente fue representada por los constructos de interés, conocimiento o una combinación de ambos (Specker et al, 2018). La experticia implicaría un alto conocimiento semántico conceptual y visual sobre artes visuales, con nociones sobre teoría e historia del arte, asociado a esquemas que permitirían mayor dominio cognitivo (clasificación estilística y elaboración sobre significados; Pelowski, Markey, Lauring, \& Leder, 2017) y comprensión basada en teoría (Bullot \& Reber, 2013).

Diversos estudios mostraron la influencia que el interés y el conocimiento previo efectivamente tienen. Van Paasschen, Bacci y Melcher (2015), por ejemplo, encontraron que diferencias en experticia -realización de actividades artísticas, interés, formación académica- no se asociaban a diferencias en aspectos emocionales básicos -valencia, activación-, pero sí a diferencias en preferencias y juicios de belleza. Algunas diferencias en preferencias -por ejemplo, por un determinado estilo artístico como el arte 
abstracto- pueden darse incluso con tiempos breves de 100ms (Pelowski et al., 2020).

Belke, Leder y Augustin (2006), a su vez, encontraron que la presencia de información explícita sobre el estilo hacía que los participantes evaluaran de manera más positiva las obras cuando tenían bajo conocimiento previo, pero no cuando eran expertos. En esta línea, Lin y Yao (2018) observaron que quienes visitaban museos con frecuencia -es decir, con mayor interés y conocimiento previo- valoraban de manera menos positiva la obras cuando eran acompañadas con información contextual, lo cual interpretaron como un efecto de distracción.

No solo las valoraciones y preferencias se ven afectadas, sino que aspectos como el procesamiento visual también varían, con las personas de menor conocimiento siendo guiadas en mayor medida por aspectos visuales superficiales (Koida, Kubo, Nishida, Shibata, \& Ikeda, 2015; Vogt \& Magnussen, 2007).

El interés en arte en general, por su parte, se vio asociado a diferencias en la forma de evaluar las obras; se observaron evaluaciones más concretas y superficiales en participantes menos interesados, mientras que en personas con mayor interés se involucraron aspectos emocionales (Afhami \& Mohammadi-Zarghan, 2018; ChamorroPremuzic \& Furnham, 2008). El grado de interés, a su vez, puede tener un impacto sobre la percepción y evaluación de la obra de arte dependiendo del contexto (Gartus, Klemer, \& Leder, 2015).

Así pues, en síntesis, diversas investigaciones en desarrollo actualmente muestran el papel de la experticia en la percepción, evaluación y vivencia de la obra de arte. Para estudiar el efecto de experticia se necesita una medición sistematizada y válida.

\section{El cuestionario de interés y conocimiento en arte de Viena (VAIAK) (Specker, Forster, Brinkmann, Boddy, Pelowski, Rosenberg, \& Leder, 2018)}

Este cuestionario se propuso como una medida adecuada de experticia en arte. Specker et al. (2018) remarcan que, aunque usualmente se los considera aspectos separados, en términos empíricos suelen confundirse interés y conocimiento $-p$. ej. preguntar por frecuencia de visitas a museos y educación en arte como un mismo indicador-. Además, los criterios para definir experticia suelen ser variables y no estar basados en teoría, como ser profesional en una institución de arte, ser artista, o haber estudiado historia del arte. Específicamente para conocimiento, Specker et al. (2018) reconocen una sola escala publicada, la Escala de Fluencia Estética (Smith \& Smith, 2006), pero que tiene la debilidad de ser una medida de auto-reporte, permeable a juicios y deseabilidad social. Por estas limitaciones, Specker et al. (2018) propusieron el Cuestionario de interés y conocimiento en arte de Viena (en adelante VAIAK por sus siglas originales). El cuestionario VAIAK está compuesto por tres partes:

-Parte A: Escala de Interés. 11 ítems de escala tipo Likert -1 a 7-, de los cuales los primeros 7 hacen referencia a aspectos de interés percibido -clases del colegio, hablar sobre arte- $\mathrm{y}$ vínculos -amigos y familia interesados en el arte-, mientras que los últimos 4 indagan frecuencia con la que se realizan actividades como ir a museos o leer 
sobre arte.

-Parte B: Escala de Conocimiento. 6 preguntas de tipo multiple choice sobre iconografía -identificación de temas o personajes representados de manera recurrente que puede darse sin conocer la obra particular, ej. San Sebastián- y técnicas artísticas. El puntaje se suma en una medida única con la parte $\mathrm{C}$.

-Parte C: Escala de Conocimiento. Implica reconocer el artista y el estilo de 10 obras que se presentan, respondiendo en un espacio en blanco, sin opciones. El puntaje se suma en una medida única con la parte $\mathrm{B}$.

El objetivo del presente trabajo es proponer una adaptación y validación del cuestionario para su uso en población argentina.

\section{Método}

\section{Participantes}

Ciento treinta y dos estudiantes de primer año de la carrera de psicología de una universidad pública argentina (edad $M=$ 22.4, $D E=6.03,74 \%$ mujeres) participaron de forma voluntaria y con consentimiento informado.

A su vez, para comprobar si el cuestionario podía identificar diferencias con una población que se estima de mayor interés y conocimiento en arte, 21 estudiantes de la carrera de historia del arte de una universidad pública argentina (edad $M=25.6, D E=5.05$, $85 \%$ mujeres) respondieron el cuestionario, de manera voluntaria y con consentimiento informado.

El proyecto fue evaluado y aprobado por un comité de ética institucional.

\section{Materiales}

Cuestionario de interés y conocimiento en arte. La traducción y adaptación del cuestionario fue discutida con cuatro miembros del equipo de investigación. Se mantuvieron todas las preguntas y se utilizaron las mismas imágenes. La presentación de imágenes fue la utilizada por Specker y colaboradores (2018), solo reemplazando la indicación "bild" por "imagen" (imágenes en https://osf.io/ckr5w/?view only=b91f3483589d48258d147bf19bf0fc7 $\overline{1}$ ). La Dra. Specker autorizó por escrito en comunicación personal, vía correo electrónico, el uso del cuestionario y estímulos. En dicha comunicación nos proporcionó la contraseña para acceder al material, cuyos detalles públicos se encuentran en https://osf.io/88d2d/, incluido el cuestionario en su idioma original.

Juicio de gusto. Se mostraron 48 imágenes de obras de arte moderno -expresionismo abstracto y arte conceptual- de artistas seleccionados en base a bibliografía (Stangos, 2000) con el criterio de que sean estáticas y mayormente en dos dimensiones -pinturas, fotografías, registro fotográfico de performance, obras con relieve, y no escultura en tres dimensiones, instalación o video- y no incluyeran palabras en otro idioma que el español. Frente a cada imagen los participantes debían indicar cuánto les gustaba la obra en una escala de 1 a 5 (1 = nada, $5=$ mucho).

\section{Procedimiento}

Luego de firmar el consentimiento informado, los participantes completaron 
los cuestionarios por escrito en una de dos modalidades: Grupal, con la presentación de imágenes proyectadas $(n=60)$, o individual, con la presentación de imágenes en pantalla $(\mathrm{n}=72)$.

En la instancia de toma grupal, luego de responder el cuestionario, se realizó la tarea de Juicio de Gusto. Se presentaron las 48 imágenes de arte moderno -ver Materialesy los participantes iban puntuando cada obra en un cuadernillo.

Adicionalmente, 21 estudiantes de historia del arte completaron voluntariamente el cuestionario VAIAK de forma remota y sin límite de tiempo, a través de un enlace que los llevó a un cuestionario online, en el que se incluyeron todas las preguntas de la parte A en una misma página $\mathrm{y}$, posteriormente, las imágenes de la parte $\mathrm{C}$ en páginas individuales con campos para completar artista y estilo.

\section{Análisis estadísticos}

Los análisis estadísticos se realizaron con el software R 3.6.2 (R Core Team, 2019). Siguiendo a Specker y colaboradores (2018) se estimó el coeficiente $\omega$ de McDonald para determinar la consistencia interna de las tres partes con el paquete psych 1.8.12 (Revelle, 2018) para los datos de los estudiantes de psicología.

Para evaluar la validez de constructo, se realizó un análisis factorial exploratorio para ver la solución factorial inicial, con el paquete psych 1.8.12 (Revelle, 2018), seguido por un análisis factorial confirmatorio con el paquete lavaan 0.6-4 (Rosseel, 2012).

Finalmente, para evaluar validez discriminante, se realizaron análisis de correlación y de diferencia de medias entre estudiantes de psicología y de historia del arte.

\section{Resultados}

\section{Escala de interés}

En la Tabla 1 se presentan los estadísticos descriptivos para los ítems de la escala de Interés (parte A). Los ítems hacen referencia al contenido, el cuestionario completo definitivo se encuentra en https://osf.io/ckr5w/?view only $=\mathrm{b} 91 \mathrm{f} 3483589 \mathrm{~d} 48258 \mathrm{~d} 147 \mathrm{bf19bf0fc71}$

El análisis factorial exploratorio con análisis paralelo -método de residuales mínimos- sugirió solo un factor latente para el conjunto de puntuaciones, y se observó un valor $K M O$ adecuado $(M S A=0.88)$. El análisis factorial confirmatorio mostró indicadores aceptables según criterios de $\mathrm{Hu}$ \& Bentler (1999), excepto para el valor del índice RMSEA: $C F I=0.922, T L I=0.902$, RMSEA $=.081\left[C I_{90}=0.053-0.108\right]$, $S R M R=0.063$. Por este motivo se puso a prueba también un modelo considerando las preguntas sobre frecuencia de actividades, y eliminando los ítems con comunalidad $<0.3$, a saber: 1.3 ("tengo varios amigos o conocidos interesados en el arte"), 1.7 ("vengo de una familia interesada en el arte") y 2.2 ("Frecuencia con la que lee libros/ revistas/catálogos sobre arte"). Este último modelo mostró un mejor ajuste $(C F I=0.963$, $T L I=0.946, R M S E A=.075\left[C I_{90}=0.027-\right.$ 0.117 ], $S R M R=0.048$ ), con una fiabilidad de $\omega$ de McDonald $=.84$ para interés percibido, $\omega$ de McDonald $=.65$ para actividades y $\omega$ de McDonald para la escala total $=.86 . \mathrm{Si}$ se diferencia la consistencia interna (escala 
Tabla 1

Estadísticos Descriptivos por Ítem para la Escala de Interés

\begin{tabular}{llccc}
\hline & $M$ & DE & Min & Max \\
1.1 Me gustaban las clases de arte & 4.63 & 1.83 & 1 & 7 \\
1.2 Me gusta hablar de arte & 4.11 & 1.92 & 1 & 7 \\
1.3 Tengo amigos o conocidos interesados & 4.11 & 2.01 & 1 & 7 \\
1.4 Me interesa el arte & 4.47 & 1.74 & 1 & 7 \\
1.5 Busco nuevas experiencias artísticas & 3.23 & 1.79 & 1 & 7 \\
1.6 Noto objetos artísticos & 3.96 & 1.80 & 1 & 7 \\
1.7 Vengo de una familia interesada & 2.43 & 1.75 & 1 & 7 \\
2.1 Frecuencia: visita a museos & 1.86 & 1.24 & 1 & 7 \\
2.2 Frecuencia: lee libros de arte & 2.16 & 1.58 & 1 & 7 \\
2.3 Frecuencia: ve imágenes de obras & 3.86 & 1.97 & 1 & 7 \\
2.4 Frecuencia: asiste a eventos de arte & 1.78 & 1.32 & 1 & 7 \\
\hline
\end{tabular}

total) entre la modalidad individual o grupal se observan respectivamente los valores $\omega$ de McDonald .84 y .88 .

\section{Escalas de Conocimiento}

En la Tabla 2 se presentan las respuestas correctas junto a la proporción de acierto para los ítems de las escalas de Conocimiento, parte B y C.

La fiabilidad para las respuestas de las escalas de Conocimiento parte $\mathrm{B}$ y C fue, respectivamente, de $\omega$ de McDonald $=.43 \mathrm{y} \omega$ de McDonald $=.84$. Debido a la baja fiabilidad se decidió no realizar análisis posteriores para la parte B. Si se diferencia entre la modalidad individual o grupal se observan respectivamente los valores $\omega$ de McDonald .60 y .53 para la parte B, y .82 y .89 para la parte $\mathrm{C}$.

La Tabla 3 muestra estadísticos descriptivos generales para las partes que resultaron confiables.

\section{Relación Interés - Conocimiento}

Al igual que Specker y cols (2018) se evaluó la relación entre los puntajes obtenidos en la escala de Interés con los de Conocimiento; al tratarse de constructos distintos pero relacionados, se espera una correlación moderada. Se empleó el coeficiente de correlación $\rho$ de Spearman debido a la cantidad de participantes y la distribución de las variables. Para el puntaje global de interés, la correlación fue significativa y positiva, $\rho=0.33, p<.05$. El resultado fue similar para los puntajes en las subescalas de Interés por separado: Interés Percibido, $\rho=0.31, p<.05$; Actividades, $\rho$ $=0.30, p<.05$.

\section{Comparación con estudiantes de historia del arte}

Se comparó el puntaje medio obtenido por los estudiantes de psicología 
74 Adaptación y validación del cuestionario de interés y conocimiento en arte de Viena...

Tabla 2

Proporción de Acierto por Ítem para las Escalas de Conocimiento

\begin{tabular}{ll} 
B1 David & 0.52 \\
B2 El nacimiento de Venus & 0.16 \\
B3 Sebastián & 0.19 \\
B4 Padre de los niños & 0.06 \\
B5 Juan el bautista & 0.40 \\
B6 Técnica mixta & 0.39 \\
C1.1 Munch & 0.08 \\
C1.2 Expresionismo & 0.04 \\
C2.1 Giotto & 0.01 \\
C2.2 Trecento & 0.05 \\
C3.1 Van Gogh & 0.44 \\
C3.2 Post-impresionismo & 0.02 \\
C4.1 Klimt & 0.06 \\
C4.2 Simbolismo & 0.01 \\
C5.1 Miguel Ángel & 0.20 \\
C5.2 Renacimiento & 0.15 \\
C6.1 Rubens & 0.01 \\
C6.2 Barroco & 0.01 \\
C7.1 Renoir & 0.01 \\
C7.2 Impresionismo & 0.05 \\
C8.1 Dalí & 0.14 \\
C8.2 Surrealismo & 0.12 \\
C9.1 Duchamp & 0.01 \\
C9.2 Dada & 0.02 \\
C10.1 Warhol & 0.16 \\
C10.2 Arte pop & 0.08 \\
\hline
\end{tabular}

con los estudiantes de historia del arte en todas las escalas.

Se tomaron para Interés tres puntajes: el de la escala total, y separada en dos factores. Para el puntaje global, el Interés fue mayor en estudiantes de historia del arte $(\mathrm{M}=43.9, \mathrm{DE}=5.7)$ que en estudiantes de psicología $(\mathrm{M}=27.9, \mathrm{DE}=9.7) ; t(41)$ $=10.59, p<.05$. Esta relación se sostiene si se consideran los puntajes de interés por separado: Interés Percibido: $U=2323, Z=$ 4.97, $p<.05, r=0.40$, Actividades: $U=$ 2599.5, $Z=6.47, p<.05, r=0.52$.

En cuanto al Conocimiento, los estudiantes de historia del arte $(\mathrm{Mdn}=15)$ también mostraron una diferencia positiva y significativa en el puntaje obtenido respecto a los estudiantes de psicología $(\mathrm{Mdn}=1) ; U$ $=2766.5, Z=7.52, p<.05, \mathrm{r}=0.61$.

\section{Relación con Gusto}

Como se explicó en el apartado de método, una submuestra $(\mathrm{n}=60)$ puntuó 48 imágenes de obras de arte moderno -expresionismo abstracto y arte conceptualen base a cuánto les gustaron.

Se realizaron correlaciones entre los puntajes obtenidos en las escales y el promedio de puntaje de Gusto. Se encontró una relación significativa y positiva con el puntaje global de Interés $(\rho=0.33, p<.05)$, al igual que considerando los puntajes por separado (Interés Percibido: $r(58)=0.27, p$

Tabla 3

Estadísticos Descriptivos para Interés (Interés Percibido y Actividades) y Conocimiento (Parte C)

\begin{tabular}{lccccc}
\hline & $M$ & $D E$ & Mdn & Min & Max \\
Interés percibido & 20.39 & 7.15 & 21.00 & 5 & 35 \\
Actividades & 7.48 & 3.52 & 6.00 & 3 & 19 \\
Conocimiento & 1.67 & 2.31 & 1.00 & 0 & 12 \\
\hline
\end{tabular}


$<.05$; Actividades $\rho=0.27, p<.05$ ).

La relación entre Conocimiento y Gusto fue de tamaño pequeña, positiva ( $\rho=$ $0.24, p=.06)$ y tendió a ser significativa.

\section{Discusión}

El objetivo de este trabajo fue traducir y evaluar la validez en el medio local del Cuestionario de Interés y Conocimiento en Arte de Viena (VAIAK) de Specker y colaboradores.

Respecto a la escala de interés, en base al análisis factorial de los datos obtenidos en este trabajo se decidió eliminar tres ítems del cuestionario original y separarlo en dos factores -Interés Percibido y Actividades-, con una fiabilidad buena en el primer caso, y aceptable en el contexto de investigación en el otro. Los puntajes obtenidos correlacionan tantojuntos como separados con las respuestas de Conocimiento y de Gusto. Y a su vez, tanto juntos como separados, los puntajes obtenidos en la muestra de estudiantes de psicología fueron significativamente menores que los de la muestra de estudiantes de historia del arte. Por estos motivos, estas subescalas pueden considerarse una medida apropiada de interés en arte visual que podrían usarse por separado o como medida global, dadas su validez de constructo según análisis factorial, y su buena fiabilidad total.

En cuanto a la parte B (conocimiento), debido a su baja fiabilidad se decidió no considerarla medida apropiada en esta adaptación. Una causa posible es que el conocimiento iconográfico es más sensible respecto a las diferencias culturales (Panofsky, 1972). Estudios posteriores podrían evaluar qué tipo de estímulos y preguntas reflejan mejor el conocimiento sobre iconografía y técnicas en esta población.

Por otro lado, la parte C (conocimiento), con una fiabilidad adecuada y correlaciones positivas con las respuestas de interés, se podría utilizar como medida de conocimiento de arte visual. A su favor cuenta además con que es una medida rápida, sencilla de administrar y puntuar, y no está basada en auto-reporte (Specker et al., 2018).

A pesar de estas conclusiones en su mayoría favorables, una limitación de la medida es que la tasa de respuestas correctas en Conocimiento fue baja y con un posible efecto piso. La medida parece no discriminar demasiado entre individuos de bajo conocimiento, pero sí cuando se los compara con expertos.

En este sentido, otra de las limitaciones fue el tamaño y la composición relativa de la muestra, sobre todo en el caso de estudiantes de historia del arte, aunque las comparaciones mostraron diferencias significativas en las medidas de conocimiento (15 de 20, comparado con 1,6 de 20 en estudiantes de psicología). En este trabajo se siguió el procedimiento original de Specker et al. (2018) con estudiantes de psicología, pero en el futuro podría evaluarse en población general, o en submuestras con posible mayor conocimiento. Quizás muestras más amplias, y con distintos niveles de rendimiento en las variables, podrían arrojar mayores ajustes en el instrumento.

Respecto a la baja proporción de aciertos en Conocimiento, otra alternativa posible sería incorporar artistas más reconocidos localmente que permitan diferenciar entre no expertos; los que tienen algún tipo de experiencia de aquellos que no 
la tienen.

En síntesis, el objetivo del presente trabajo fue adaptar el VAIAK para ser aplicado en el contexto local. Si bien estudios futuros deberían ampliar y diversificar la muestra tomada en este primer abordaje, se han obtenido evidencias de la fiabilidad y validez de las escalas de Interés, y de una parte de la escala de Conocimiento. Así se cuenta con una medida útil del interés y el conocimiento previo sobre arte visual, para implementar en futuras investigaciones sobre la relevancia de estas variables en distintos aspectos del procesamiento y la experiencia estética.

\section{Referencias}

Afhami, R., \& Mohammadi-Zarghan, S. (2018). The Big Five, aesthetic judgement styles, and art interest. Europe's Journal of Psychology, 14(4), $\quad 764 . \quad$ https://dx.doi. org/10.5964\%2Fejop.v14i4.1479.

Arnheim, R. (1965). Art and visual perception: A psychology of the creative eye. Berkeley, CA: Univ of California Press.

Belke, B., Leder, H., \& Augustin, D. (2006). Mastering style. Effects of explicit style-related information, art knowledge and affective state on appreciation of abstract paintings. Psychology Science, 48(2), 115. Recuperado de https://psycnet.apa.org/ record/2006-10718-003.

Bullot, N. J., \& Reber, R. (2013). The artful mind meets art history: Toward a psycho-historical framework for the science of art appreciation. Behavioral and Brain Sciences, 36(2), 123137. https://doi.org/10.1017/ S0140525X12000489.

Chamorro-Premuzic, T., \& Furnham, A. (2008). Personality, intelligence and approaches to learning as predictors of academic performance. Personality and Individual Differences, 44(7), 15961603. https://doi.org/10.1016/j. paid.2008.01.003.

Fancourt, D., \& Finn, S. (2019). What is the evidence on the role of the arts in improving health and well-being?. Copenhagen: WHO Regional Office for Europe.

Fechner, G. T. (1876). Vorschule der ästhetik. Leipzig: Breitkopf \& Härtel.

Gartus, A., Klemer, N., \& Leder, H. (2015). The effects of visual context and individual differences on perception and evaluation of modern art and graffiti art. Acta Psychologica, 156, 64-76. https://doi.org/10.1016/j. actpsy.2015.01.005.

Hu, L. T., \& Bentler, P. M. (1999). Cutoff criteria for fit indexes in covariance structure analysis: Conventional criteria versus new alternatives. Structural Equation Modeling: A Multidisciplinary Journal, 6(1), 1-55. https://doi. org/10.1080/10705519909540118.

Koida, N., Kubo, T., Nishida, S., Shibata, T., 
\& Ikeda, K. (2015). Art expertise reduces influence of visual salience on fixation in viewing abstract paintings. PloS One, 10, e0117696. https://doi.org/10.1371/ journal.pone.0117696.

Kou, X., Konrath, S., \& Goldstein, T. R. (2019). The relationship among different types of arts engagement, empathy, and prosocial behavior. Psychology of Aesthetics, Creativity, and the Arts. Advance online publication. https://doi. org/10.1037/aca0000269.

Lin, F., \& Yao, M. (2018). The impact of accompanying text on visual processing and hedonic evaluation of art. Empirical Studies of the Arts, 36(2), 180-198. https://doi. org $/ 10.1177 / 0276237417719637$.

Meskin, A., Robson, J., Ichino, A., Goffin, K., \& Monseré, A. (2018). Philosophical aesthetics and cognitive science. Wiley Interdisciplinary Reviews: Cognitive Science, 9(1), e1445. https://doi.org/10.1002/wcs.1445.

Ortlieb, S. A., \& Carbon, C. C. (2019). Kitsch and perception: towards a new 'aesthetic from below'. Art \& Perception, 7(1), 1-26. https://doi. org/10.1163/22134913-00001091.

Panofsky, E. (1972). Studies in iconology: Humanistic themes in the art of the Renaissance. New York, NY: Harper \& Row.

Pelowski, M., Cabbai, G., Brinkmann, H., Mikuni, J., Hegelmaier, L. M., Forster, M., Rosenberg, R., \& Leder, H. (2020). The kitsch switch-or (when) do experts dislike Thomas Kinkade art? A study of time-based evaluation changes in top-down versus bottom-up assessment. Psychology of Aesthetics, Creativity, and the Arts. Advance online publication. https://doi. org/10.1037/aca0000302.

Pelowski, M., Markey, P. S., Forster, M., Gerger, G., \& Leder, H. (2017). Move me atonish me... delight my eyes and brain: The Vienna integrated model of topdown and bottom-up processes in art perception (VIMAP) and corresponding affective, evaluative, and neurophysiological correlates. Physics of Life Reviews, 21, 80125. https://doi.org/10.1016/j. plrev.2017.02.003.

Pelowski, M., Markey, P. S., Lauring, J. O., \& Leder, H. (2016). Visualizing the impact of art: An update and comparison of current psychological models of art experience. Frontiers in human neuroscience, 10, 160. https://doi. org/10.3389/fnhum.2016.00160.

$\mathrm{R}$ Core Team (2019). $R$ : A language and environment for statistical computing. $R$ Foundation for Statistical Computing, Vienna, Austria. Available online at https:// www.R-project.org/

Revelle, W. (2018) psych: Procedures for Personality and Psychological Research, Northwestern University, Evanston, Illinois, USA, https://CRAN.R-project.org/ package $=$ psych Version $=1.8 .12$.

Smith, L. F., \& Smith, J. K. (2006). The 
78 Adaptación y validación del cuestionario de interés y conocimiento en arte de Viena...

nature and growth of aesthetic fluency. In P. Locher, C. Martindale, \& L. Dorfman (Eds.), Foundations and Frontiers in Aesthetics. New directions in aesthetics, creativity and the arts (p. 47-58). Amityville, NY: Baywood.

Specker, E., Forster, M., Brinkmann, H., Boddy, J., Pelowski, M., Rosenberg, R., \& Leder, H. (2018). The Vienna Art Interest and Art Knowledge Questionnaire (VAIAK): A unified and validated measure of art interest and art knowledge. Psychology of Aesthetics, Creativity, and the Arts. Advance online publication. https:// doi.org/10.1037/aca0000205.

Stangos, N. (2000). Conceptos del arte moderno: del fauvismo al posmodernismo. Barcelona: Destino.
Rosseel, Y. (2012). lavaan: An R Package for Structural Equation Modeling. Journal of Statistical Software, 48(2), 1-36. http://dx.doi. org/10.18637/jss.v048.i02.

van Paasschen, J., Bacci, F., \& Melcher, D. (2015). The influence of art expertise and training on emotion and preference rating for representational and abstract artworks. PLoS One, 10(8): e0134241. https:// dx.doi.org/10.1371\%2Fjournal. pone. 0134241 .

Vogt, S., \& Magnussen, S. (2007). Expertise in pictorial perception: eyemovement patterns and visual memory in artists and laymen. Perception, 36(1), 91-100. https://doi.org/10.1068/p5262. 